\title{
MICROSTRUCTURE VARIATION EFFECTS ON ROOM TEMPERATURE FATIGUE THRESHOLDS AND CRACK PROPAGATION IN UDIMET 720Li Ni-BASE SUPERALLOY.
}

\author{
H.T. Pang*, P.A.S. Reed
}

Materials Research Group, School of Engineering Sciences, University of Southampton, Highfield, Southampton SO17 1BJ, United Kingdom.

\begin{abstract}
An assessment of the effects of microstructure on room temperature fatigue threshold and crack propagation behaviour has been carried out on microstructural variants of U720Li, i.e. as-received U720Li, U720Li-LG (large grain variant) and U720Li-LP (large intragranular coherent $\gamma^{\prime}$ variant). Fatigue tests were carried out at room temperature using a $20 \mathrm{~Hz}$ sinusoidal cycling waveform at an Rratio=0.1. U720Li-LG showed the highest threshold $\Delta K\left(\Delta K_{t h}\right)$, whilst U720Li-LP showed the lowest $\Delta K_{t h}$ value. U720Li-LP also showed higher crack growth rates in the near-threshold regime and at high $\Delta K$ (although at higher $\Delta K$ levels the difference was less marked). Crack growth rates of U720Li and U720Li-LG were relatively similar both in the near-threshold and high $\Delta K$ regime. The materials showed crystallographic stage I type crack growth in the near-threshold regime, with U720Li showing distinct crystallographic facets on the fracture surface while U720Li-LG and U720Li-LP showed mostly microfacets and a lower proportion of large facets. At high $\Delta K$, crack growth in the materials becomes flat and featureless indicative of stage II type crack growth. The observed performance of the materials is rationalised in terms of their microstructural characteristics. Enhanced room temperature fatigue threshold and long crack growth resistance are seen for larger grained materials due to increased extrinsic crack growth resistance contributions from crack closure. Differences in heterogeneity of deformation behaviour in this set of material variants appear to give approximately equivalent intrinsic crack growth resistance at room temperature due to the respective effects of each deformation behaviour on intrinsic crack growth resistance.
\end{abstract}

Keywords: Udimet 720Li, microstructural effects, fatigue threshold, fatigue crack propagation.

\subsection{INTRODUCTION}

Turbine disc operating conditions are extremely severe due to repeated load cycling where one major loading cycle typically corresponds to start-up and climb followed by descent and shutdown. During cruise, prolonged loading at elevated temperatures is combined with exposure to hot corrosive gases.

\footnotetext{
* Now at Dept. of Materials Science \& Metallurgy, University of Cambridge, Pembroke Street, Cambridge CB2 3QZ, UK
} 
Under these conditions, damage to components is severe with combinations of fatigue, creep, oxidation and corrosion taking place. Nickel-base superalloys have conventionally been considered as candidate turbine disc materials as they have a good balance of the properties critical to turbine disc operation, i.e. a combination of fatigue, creep, corrosion and oxidation resistance.

Fatigue in turbine discs constitutes one of the major causes of component life reduction. When coupled with creep and oxidation experienced under operating conditions, the synergistic effects of these damage mechanisms are likely to give increased crack growth rates and hence accelerated reduction in component life compared to that caused by individual damage mechanisms. In order to assess these interactions it is important to consider the individual damage mechanisms in detail. Low temperature fatigue behaviour in these systems is worthy of study as it has operational significance for lower temperature areas such as the bore and it provides an important basis for understanding transitions in crack growth mechanisms that can lead to optimised alloy design.

The fatigue crack growth process is a complicated function of a number of variables, including stress intensity, load ratio, temperature, frequency, hold time and environment. In general, the crack growth process can be grouped into two categories, i.e. cycle-dependent crack growth and time-dependent crack growth. Note that these two categories of crack growth are not mutually exclusive, i.e. mixed cycle-dependent and time-dependent crack growth may be seen in practice. Generally, cycledependent behaviour is favoured by low temperatures, high cycling frequencies and inert environments, whereas time-dependent behaviour is prevalent at high temperatures, low cycling frequencies, and in oxidising or corrosive environments $[1,2]$. During cycle-dependent crack growth, the crack growth rate per cycle, $d a / d N$, is controlled by characteristics of the loading cycle, e.g. $\Delta K$ and load ratio. Temperature has little effect on the crack propagation process except as it affects the yield strength and modulus of the material. Other test parameters, e.g. frequency, hold-time and environment, which essentially affect time-dependent processes, will also have little effect on the cycle-dependent crack growth process. Cycle-dependent crack growth is usually characterised by a relatively flat transgranular crack path, sometimes with evidence of striations. Time-dependent crack growth behaviour is seen when time-dependent damage mechanisms, i.e. creep and environment effects (usually along grain boundaries) contribute significantly to the fatigue crack growth process. Time-dependent crack growth is therefore typically characterised by an intergranular fracture surface.

In the cycle-dependent regime, fatigue crack growth can be divided into three distinct crack growth regimes as $d a / d N$ varies with $\Delta K$. Regime I is the near-threshold crack growth regime, where crack growth is extremely slow, and below the threshold $\Delta K\left(\Delta K_{t h}\right)$ no crack growth will occur. The $\Delta K_{t h}$ 
value is therefore to some extent an indication of the intrinsic resistance of the material to fatigue processes (although it can be highly dependent on the R-ratio used due to crack closure effects [3]). Crack growth behaviour in the near-threshold regime has been noted to be highly sensitive to microstructure, where slip-band decohesion processes (sometimes called quasi-Stage I crack growth) can give rise to distinct crystallographic facets on the fracture surface. Regime II denotes the region of stable crack growth (known as the Paris regime) where $d a / d N$ relates to $\Delta K$ according to the Paris equation:

$$
\frac{d a}{d N}=A(\Delta K)^{n}
$$

where $A$ and $n$ are material constants. Crack growth behaviour in this regime is usually fairly insensitive to microstructure, and a flat transgranular Stage II type crack propagation mechanism predominates. Regime III denotes the accelerated crack growth region under high $\Delta K$ leading to final failure, where monotonic static failure modes contribute to crack growth. The proportion of life taken up by Regime III is however very small for most applications, hence most focus is usually placed on understanding the crack growth resistance at lower $\Delta K$ levels, i.e. Regime I and Regime II.

This paper reports part of a study carried out to elucidate the effects of microstructure on fatigue crack growth behaviour in powder metallurgy (PM) nickel-base turbine disc superalloy Udimet 720Li (U720Li). In particular this paper concentrates on the effects of microstructure on fatigue threshold and long crack propagation at room temperature. Other complementary papers $[4,5,6]$ detail the effects of microstructure on long fatigue crack growth at elevated temperatures in these (and comparator) alloys, and two further papers focus on fatigue crack initiation and early crack growth behaviour in these alloys at room temperature [7] and elevated temperature [8]. Data from these other publications is referenced for comparison purposes in this paper, where appropriate.

\subsection{MATERIAL}

The materials used in this study were based on the powder metallurgy (PM) turbine disc material U720 Li, which was studied in its as-received form and in two microstructural variants produced by further heat treatments: U720Li-LG (large grain variant) and U720Li-LP (large intragranular coherent $\gamma^{\prime}$ precipitate variant). Nickel-base superalloys consist of a f.c.c. $\gamma$ matrix strengthened by coherent $\gamma^{\prime}$ precipitates. Primary $\gamma^{\prime}$ precipitates which are incoherent with the $\gamma$ matrix may reside at grain boundaries, these are typically $\gamma^{\prime}$ remaining from the original powder process which may not have dissolved during solution heat treatment, and so may be present depending on whether a sub-solvus or super-solvus solution heat treatment is used. They typically act to pin the grain boundaries during a 
sub-solvus heat treatment. The composition of U720Li is given in Table 1 and the heat treatment routes used for each material are listed in Table 2. Microstructural variants of U720Li were produced by increasing solution temperature (to increase grain size) and by slowing the cooling rate from solution temperature (to increase coherent $\gamma^{\prime}$ precipitate size) [6].

\subsection{EXPERIMENTAL}

\subsection{Microstructural characterisation}

Electrolytic etching with $10 \%$ orthophosphoric acid in water revealed the microstructures of the test materials as shown in Figure 1. Primary $\gamma^{\prime}$ precipitates (white) can be distinguished from the $\gamma$ matrix and appear to reside on the $\gamma$ grain boundaries. Grain size and primary $\gamma^{\prime}$ precipitates size and volume fraction were determined using image analysis, where an automatic image analysis software Carl Zeiss KS300 (by Carl Zeiss Vision) was used to quantify various parameters (e.g. equivalent circular diameter, ferets, area) of the grains and primary $\gamma^{\prime}$ precipitates. Coherent $\gamma^{\prime}$ characterisation was carried out by analysing carbon extraction replicas on a JEOL 2000FX TEM operating at 200kV. Subsequent image analysis of the TEM images yielded the size characteristics of coherent secondary and tertiary $\gamma^{\prime}$ precipitates. The Vickers hardness values of the three materials at room temperature were also assessed.

\subsection{Mechanical testing}

Long crack fatigue tests were carried out at room temperature on an Instron $8802( \pm 10 \mathrm{kN}$ capacity $)$ servohydraulic testing machine. All tests were conducted in 3-point bend under load control using an R-ratio of 0.1 and a $20 \mathrm{~Hz}$ sinusoidal waveform. A span of $20 \mathrm{~mm}$ was used in all tests. Test samples were $12.5 \mathrm{~mm} \times 12.5 \mathrm{~mm}$ square section SENB specimens with a $60^{\circ}$ starter notch as shown in Figure 2. Crack lengths were monitored using a 2-point potential drop (P.D.) method as per ASTM 647 calibrated for the notch type used. P.D. detection wires were spot-welded at opposite ends of the notch, one on each side, and current supply bolts were attached to both ends of the specimens. Precracking was started at a constant $\Delta K$ of $\sim 20 \mathrm{MPa} \sqrt{\mathrm{m}}$ for approximately $0.5 \mathrm{~mm}$ of crack growth followed by growth through four monotonic plastic zone sizes, to ensure $d a / d N$ values obtained were not affected by start-up effects from the notch. Load shedding (in 10\% decrements after growth through four monotonic plastic zone sizes at each constant $\Delta K$ level) was then continued until crack growth rates reached the order of $10^{-12} \mathrm{~m} / \mathrm{cycle}$. This was followed by growth out under constant load condition, i.e. increasing $\Delta K$ as the crack grew, thus the range of $d a / d N$ versus $\Delta K$ behaviour was captured. After testing, the crack lengths corresponding to the notch, threshold region and final fracture were measured. A linear correction factor was used to correlate the crack lengths obtained from P.D. measurements (and the associated calibration) and the actual crack lengths observed on the 
fracture surface. This was to correct changes in the P.D. measurement due to differences in temperature during calibration and testing, as well as to correct possible electrical closure effects during the tests. The P.D. data measured and logged during the tests was then converted to corresponding corrected crack length and load cycle information. The crack length and load cycle information were then converted into crack growth rate $d a / d N$ and the corresponding stress intensity factor range $\Delta K$. The crack growth rates were calculated using the secant method.

Detailed fractographic work to examine the fracture surfaces was carried out in a JEOL JSM-6500 FEG-SEM operating at an accelerating voltage of $10 \mathrm{kV}$. The fracture surfaces were also sectioned longitudinally (in the direction of crack growth), mounted, polished and etched to enable the crack path to be observed with respect to the microstructure near the fracture surface in the SEM.

\subsection{RESULTS}

The microstructural characteristics of the materials are presented in Table 3, which also shows the hardness values of the materials obtained at room temperature. U720Li-LG has the largest grain size and lowest volume fraction of primary $\gamma^{\prime}$, whilst U720Li-LP has the largest secondary and tertiary $\gamma^{\prime}$ precipitates. The following room temperature hardness ranking was observed: U720Li > U720Li-LG $>$ U720Li-LP.

The growth out curves of the room temperature long crack tests are shown in Figure 3 . The $\Delta K_{t h}$ values of the materials are presented in Table 4. From Figure 3 it can be seen that in the nearthreshold and high $\Delta K$ region, U720Li and U720Li-LG have very similar crack growth rates. U720LiLP has significantly higher crack growth rates than other materials in the near-threshold $\Delta K$ region. At higher $\Delta K$ the crack growth rates of U720Li-LP converge close to the crack growth rates of other materials but are still marginally higher compared to other materials. Comparing the $\Delta K_{t h}$ in Figure 3 and Table 4, U720Li and U720Li-LG have very similar $\Delta K_{t h}$ values whilst U720Li-LP has significantly lower $\Delta K_{t h}$. U720Li-LG, which has largest grains, exhibits the highest $\Delta K_{t h}, \mathrm{U} 720 \mathrm{Li}$ which has an intermediate grain size, has a $\Delta K_{t h}$ between that of U720Li-LG and U720Li-LP. Figures 4-6 show the fracture surfaces and sectioned fracture surfaces of the tests as observed in the SEM.

In the near-threshold region, crystallographic facets corresponding to stage I crack growth were observed on the fracture surfaces of all test materials. The degree of crystallographic faceting was however somewhat different in the materials. At near-threshold $\Delta K$, the most distinct crystallographic facets indicative of highly crystallographic crack growth were observed in U720Li (Figures 4a-d), while U720Li-LG (Figures 5a-d) and U720Li-LP (Figures 6a-d) exhibit lower but approximately 
similar degrees of crystallographic faceting, most of which are micro-facets and a low proportion of large facets. The degree of faceting decreased with increasing $\Delta K$ in all the materials, and faceted crack growth was not observed at high $\Delta K$ where a flatter fracture surface was observed, indicative of increasing stage II crack growth. Evidence of fretting was also observed on the fracture surfaces in the near-threshold crack growth region. The fretting damage noted in U720Li-LG and U720Li-LP (Figures 5a and 6a) was relatively similar while in U720Li (Figure 4a) the degree of fretting damage appeared to be minimal with crystallographic facets appearing undamaged.

To shed further light on near-threshold crack growth behaviour, an assessment of the effects of crack deflection on near-threshold crack growth was carried out. The influence of crack deflection on crack tip driving force during near-threshold crack growth could be significant. The crack profiles of the near-threshold crack growth region as shown in Figure 7 were obtained from the sectioned fracture surface fractographs obtained at low magnification in the SEM (Figures 4b, 5b, 6b). The angle of crack deflection and the deflected actual crack path length at each deflection was measured from these crack profiles. The ratio of actual crack path length to projected crack length was plotted in Figure 8a as a function of the projected crack length in the crack growth direction. Large scatter in the ratio of actual to projected crack length was noted for all materials, except for U720Li-LG which appears to consistently have a ratio close to 1 .

The effects of crack deflection on local elastic crack tip driving force $(k)$ can be calculated using the solutions provided by Bilby et al [9]. The values of the local stress intensities $k_{l}$ and $k_{2}$ ahead of the crack tip as shown in Figure $8 \mathrm{~b}$ can be related to the remotely applied $K_{I}$ and deflection angle $\alpha$ according to the following equations [9]:

$$
\begin{array}{ll}
k_{1}=\frac{1}{4}\left(3 \cos \frac{\alpha}{2}+\cos \frac{3 \alpha}{2}\right) K_{I} & \text { Eqn. (2) } \\
k_{2}=\frac{1}{4}\left(\sin \frac{\alpha}{2}+\sin \frac{3 \alpha}{2}\right) K_{I} & \text { Eqn. (3) }
\end{array}
$$

The effective $K\left(K_{\text {eff }}\right)$ is then defined as:

$$
K_{e f f}=\sqrt{k_{1}^{2}+k_{2}^{2}} \quad \text { Eqn. (4) }
$$

The effects of crack deflection in the near-threshold crack growth region are plotted as a function of $\Delta K_{\text {eff }}$ against the projected crack length in the crack growth direction in Figure 8c. Large scatter in the 
$\Delta K_{\text {eff }}$ is noted for all materials, except for U720Li-LG which shows lower scatter, consistent with the observations of Figure 8a.

\subsection{DISCUSSION}

\subsection{Microstructure}

The grain sizes and primary $\gamma^{\prime}$ volume fractions of the test materials are generally consistent with the solution temperatures used. Higher solution temperatures for a given alloy chemistry are expected to cause dissolution of grain boundary primary $\gamma^{\prime}$ precipitates, leading to grain growth at the expense of other grains and primary $\gamma^{\prime}$ precipitates, and hence larger grains and lower primary $\gamma^{\prime}$ volume fractions will be observed. Larger grains were noted in U720Li-LG compared to U720Li, and higher primary $\gamma^{\prime}$ volume fractions were noted in U720Li and U720Li-LP compared to U720Li-LG, consistent with the solution temperatures used during heat treatment. Comparing the grain sizes of U720Li and U720LiLP, larger grains were noted in U720Li-LP although similar solution temperature was used for both materials; this may be due to variation in the temperatures experienced across the original disc forging.

In terms of coherent $\gamma^{\prime}$ precipitates, nucleation of secondary and tertiary $\gamma^{\prime}$ occurs at different stages during cooling from solution temperature. Nucleation of secondary $\gamma^{\prime}$ precipitates occurs at temperatures very near the solution temperature due to changes in equilibrium $\gamma^{\prime}$ fraction as temperature is lowered as shown in Figure 9 [10], i.e. the matrix becomes supersaturated with $\gamma^{\prime}$ forming elements when the disc forging is cooled from solution temperature. Some growth of the nucleated secondary $\gamma^{\prime}$ precipitates is also expected to occur during cooling from solution temperature. Tertiary $\gamma^{\prime}$ precipitates nucleate at lower temperatures during cooling when the diffusion rate of solutes are slow and secondary $\gamma^{\prime}$ precipitates are unable to grow fast enough to keep supersaturation of $\gamma^{\prime}$-forming elements in matrix low, and at a critical undercooling the driving force for nucleation is sufficient to allow nucleation of tertiary $\gamma^{\prime}$ to occur [11]. Growth of tertiary $\gamma^{\prime}$ precipitates is however expected to be limited as the temperature is likely to be too low to allow intensive solute diffusion when tertiary $\gamma^{\prime}$ precipitates are nucleated [12]. The cooling rate during cooling from solution temperature has been indicated to be the controlling parameter governing the size of coherent $\gamma^{\prime}$ precipitates [12]. Higher cooling rates have been associated with a finer size and lower volume fraction of secondary $\gamma^{\prime}$ precipitates, and vice versa for lower cooling rates. Higher cooling rates give greater $\gamma^{\prime}$ nucleation site frequency and in addition also limit the growth of secondary $\gamma^{\prime}$ precipitates as there is less time for the diffusional processes necessary for the growth of $\gamma^{\prime}$ precipitates. Lower cooling rates are expected to give a more uniform nucleation of $\gamma^{\prime}$ precipitates throughout the material and allow a greater degree of precipitate growth during cooling from solution temperature. The 
tertiary $\gamma^{\prime}$ size has been indicated to be relatively insensitive to the cooling rate used $[11,12]$. Further coarsening of the nucleated tertiary $\gamma^{\prime}$ precipitates (regardless of the cooling rates used after solution treatment) occurs during the double ageing treatment stages. The secondary $\gamma^{\prime}$ size is believed to be unaffected during the ageing process, thus the main microstructural change during ageing is the growth of tertiary $\gamma^{\prime}$ precipitates $[11,12]$. The non-equilibrium $\gamma^{\prime}$ volume fraction provides the driving force for coarsening of tertiary $\gamma^{\prime}$ precipitates, with the volume fraction of $\gamma^{\prime}$ actually increasing during ageing.

The secondary $\gamma^{\prime}$ sizes of the U720Li variants in this study reflected the cooling rates used. U720Li was oil quenched, while U720Li-LG was air-cooled and U720Li-LP was insulated air-cooled. The asreceived U720Li material (which has experienced the highest cooling rate) has the smallest secondary $\gamma^{\prime}$ size, while U720Li-LG which has been air-cooled (i.e. a lower cooling rate compared to U720Li) has larger secondary $\gamma^{\prime}$ precipitates compared to U720Li. U720Li-LP, which has experienced the lowest cooling rate, has the largest secondary $\gamma^{\prime}$ precipitates amongst the U720Li variants. Although the tertiary $\gamma^{\prime}$ precipitates are expected to be insensitive to the cooling rates used during cooling from solution temperature [11], the results of this study appear to suggest otherwise. After the ageing treatment, the material which experienced higher cooling rates from solution temperature also has finer tertiary $\gamma^{\prime}$ size. As all the materials received the same two-stage ageing treatment, the degree of tertiary $\gamma^{\prime}$ precipitate growth is expected to be relatively similar. This suggests that the size of tertiary $\gamma^{\prime}$ nuclei after cooling from solution temperature may be markedly different in the materials, and is likely to be smaller for materials which have undergone faster cooling, similar to the effect of cooling rate on secondary $\gamma^{\prime}$ precipitate size.

An estimation of the volume fractions of secondary and tertiary $\gamma^{\prime}$ precipitates in the materials was carried out by referring to a study by Mao et al on U720Li [12] where the volume fraction of secondary $\gamma^{\prime}$ was noted to attain a relatively constant value at approximately $900^{\circ} \mathrm{C}$. This is also likely to apply to the materials in the current study where the stable volume fraction of secondary $\gamma^{\prime}$ may be assumed to be formed in the materials at $900^{\circ} \mathrm{C}$. By assuming that the primary and secondary $\gamma^{\prime}$ volume fractions make up the equilibrium volume fraction of $\gamma^{\prime}$ expected at $900^{\circ} \mathrm{C}$, the volume fraction of secondary $\gamma^{\prime}$ in the materials can be calculated by subtracting the observed volume fraction of primary $\gamma^{\prime}$ precipitates (from image analysis of room temperature samples) from the total volume fraction of $\gamma^{\prime}$ expected at $900^{\circ} \mathrm{C}$. Similarly, by assuming that any further $\gamma^{\prime}$ in the material forms tertiary $\gamma^{\prime}$ precipitates during ageing and on cooling from the ageing temperature, the volume fraction of tertiary $\gamma^{\prime}$ can be calculated by subtracting the volume fraction of $\gamma^{\prime}$ expected at $900^{\circ} \mathrm{C}$ from the volume fraction of $\gamma^{\prime}$ expected at $250^{\circ} \mathrm{C}$ (which is the temperature at which the maximum volume 
fraction of $\gamma^{\prime}$ is expected). In reality, the volume fraction of tertiary $\gamma^{\prime}$ in the materials will be slightly lower than the calculated value as the relatively low temperature especially during cooling from the final ageing temperature and possible rapid cooling may suppress diffusional growth of tertiary $\gamma^{\prime}$ precipitates so the equilibrium volume fraction of $\gamma^{\prime}$ expected at $250^{\circ} \mathrm{C}$ may not be attained. The results of the calculations of secondary and tertiary $\gamma^{\prime}$ volume fractions for the materials in the current study are shown in Table 3. Note that the volume fractions of $\gamma^{\prime}$ expected at $900^{\circ} \mathrm{C}$ and $250^{\circ} \mathrm{C}$ were obtained from Thermo-calc predictions carried out at the University of Cambridge, the results of which are shown in Figure 9.

The coherent $\gamma^{\prime}$ distribution in the materials is expected to affect slip character, although it seems likely that the fine tertiary $\gamma^{\prime}$ will have the greatest effect on the degree of slip planarity. The planarity of slip has been assessed in other alloys, e.g. the dislocation-precipitate cutting to bowing transition was observed in Waspaloy [13] and Nimonic 90 [14] to be 20-25nm, so it seems likely that precipitate cutting is occurring in all three U720Li variants in the current study, but possibly there may be a combination of bowing and cutting in U720Li-LP. Based on this, the planarity of slip is believed to be greatest in U720Li-LG due to the combination of larger grains (hence increased slip band length and possible dislocation pile-up) and relatively fine coherent $\gamma^{\prime}$ distributions, followed by U720Li and then U720Li-LP.

The ranking of the hardness values of the materials (U720Li > U720Li-LG > U720Li-LP) reflects the flow strength ranking at room temperature. The strength of these materials is primarily governed by grain size and the size and volume fraction of coherent $\gamma^{\prime}$ precipitates. The Hall-Petch relationship predicts that reduction in the number of grain boundaries acting as barriers to slip will reduce the strength of larger grained materials. However the volume fraction of strengthening (coherent) $\gamma^{\prime}$ precipitates may also play an important role, with higher volume fractions of $\gamma^{\prime}$ giving higher strength levels. For a given volume fraction of coherent $\gamma^{\prime}$, larger $\gamma^{\prime}$ sizes may lead to larger mean distances between precipitates and hence less effective blocking of dislocations (possibly allowing by-passing or looping of precipitates if mean precipitate spacing become large enough). The three U720Li variants examined do not explicitly vary one of these microstructural variants whilst keeping the others constant, partly because some of the variables are correlated (e.g. volume fraction of coherent $\gamma^{\prime}$ and grain size). However it seems as though the larger grain size of U720Li-LG (and to a lesser extent of U720Li-LP) has led to a decrease in strength $c f$. U720Li, which has been exacerbated in the case of U720Li-LP by larger coherent $\gamma^{\prime}$ sizes, hence this material shows the lowest strength. 
Stage I type crack growth occurs in the near-threshold crack growth regime in the room temperature long crack tests. Stage I crack growth was also observed in these materials at short crack lengths immediately after crack initiation in corresponding short crack tests [7]. Stage I crack growth corresponds to crack growth where the zone of plastic deformation surrounding the crack tip is confined within a few grains, and crack growth occurs predominantly along slip bands in the slip system experiencing the maximum resolved shear. This gives rise to a zig-zag crack path and a highly facetted fracture surface as the crack path changes with slip systems going from grain to grain. In the long crack tests, although the defect size is physically large, the low applied loads meant that the crack tip plastic zones are much smaller than the grain size, hence multiple co-operative slip is not operational and crack propagation occurs in a stage I manner. Stage I crack growth is generally considered to be highly sensitive to the local microstructure e.g. grain boundaries (especially at small crack lengths) and other extrinsic factors which may be enhanced due to the crack growth mode (e.g. crack closure, crack tip shielding, etc).

At longer crack lengths, the size of the plastically deformed zone ahead of the crack tip increases to encompass several grains, and slip systems in several grains are activated. At this point, crack growth is able to occur through simultaneous or co-operative slip along these multiple slip systems, i.e. Stage II crack growth. Stage II crack growth is characterised by a relatively flat and featureless transgranular fracture surface. Stage II crack growth is less sensitive to local microstructure compared to Stage I crack growth and the crack samples the averaged microstructural characteristics of the material during Stage II crack growth.

\subsection{Room temperature short crack-long crack fatigue comparison}

A general comparison of room temperature short crack and long crack growth behaviour is made by comparing the short crack [8] and long crack growth curves for each material as shown in Figures 10 a-c. In the short crack tests, initially scattered Stage I short crack growth behaviour under the influence of local microstructure (e.g. grain boundaries and primary $\gamma^{\prime}$ precipitates giving rise to temporary crack arrests) is observed followed by a transition to stage II crack growth [8]. In the long crack tests, Stage I near-threshold long crack growth rates are less sensitive to the local microstructure, due to the longer crack front sampling more microstructure, but is heavily influenced by extrinsic factors such as crack closure at low and near-threshold $\Delta K$ and again a transition to stage II crack growth occurs at higher $\Delta K$. Convergence of the two curves will therefore indicate the point of transition to structure-insensitive crack growth, i.e. a transition from stage I to stage II crack growth. In the current work, the short crack growth curves and long crack growth curves do not show a very distinct point of convergence, although the crack growth curves were noted to merge at higher 
$\Delta K$. Although no truly distinct points of convergence were noted, an approximation can be made from the plots. The approximate $\Delta K$ values at the point of convergence between the short crack growth curves and the long crack growth curves, $\Delta K_{\text {conv }}$ are presented in Table 5. A comparison of the monotonic and cyclic plastic zone size corresponding to $\Delta K_{\text {conv }}$ and the grain size of the material is also presented in Table 5. The plastic zone size is estimated using Irwin's approximation [15].

Stage I crack growth is thought to occur when plasticity is totally contained within the grains, (i.e. when the monotonic plastic zone size is less than the grain size) whilst stage II crack growth occurs when the cyclic plastic zone size exceeds the grain size, between these two stages a mixed stage I/stage II crack growth mode will be observed. At the estimated $\Delta K_{\text {conv }}$, predominantly stage II crack growth does indeed appear to be occurring as the monotonic plastic zone has encompassed at least 3 grains in the materials and the cyclic plastic zone is approximately equal to the grain size. This is confirmed by fracture surface observations in Figures 4-6 where the fracture surfaces at high $\Delta K(\sim$ $\Delta K_{\text {conv }}$ ) show relatively flat transgranular crack growth with little evidence of crystallographic facets.

\subsection{Room temperature and $650^{\circ} \mathrm{C}$ cycle-dependent long crack growth comparison}

A comparison of room temperature long crack growth behaviour in air and elevated temperature long crack growth behaviour at $650^{\circ} \mathrm{C}$ in vacuum for each material is shown in Figure 11 . Note that the data compared are from constant load growth-out (increasing $\Delta K$ ) conditions. The elevated temperature long crack tests at $650^{\circ} \mathrm{C}$ in vacuum were carried out at much lower frequencies using a 1-x-1-1 trapezoidal loading waveform, where $\mathrm{x}$ was 1 or 20 seconds [4].

The crack growth behaviour of $\mathrm{U} 720 \mathrm{Li}$ and its variants at $650^{\circ} \mathrm{C}$ in vacuum is believed to be cycledependent due to the absence of significant dwell time effects - observed when comparing $650^{\circ} \mathrm{C} 1-1$ 1-1 and 1-20-1-1 crack growth rates. A comparison between the room temperature crack growth rates in air, which are cycle-dependent and the crack growth rates at $650^{\circ} \mathrm{C}$ in vacuum should therefore reveal the effects of temperature on cycle-dependent crack growth behaviour. The cycle-dependent nature of crack growth at both temperatures $\left(20^{\circ} \mathrm{C}\right.$ and $\left.650^{\circ} \mathrm{C}\right)$ is enforced by the similarity in the slope of the room temperature and $650^{\circ} \mathrm{C}$ crack growth curves, as well as by the similar transgranular stage II crack growth fracture surface observed at both temperatures [4]. Both observations suggest similar crack growth mechanism at room temperature and $650^{\circ} \mathrm{C}$. The crack growth rates at $650^{\circ} \mathrm{C}$ in U720Li and variants are generally about one decade higher than the room temperature crack growth rates. This is believed to reflect the increased dislocation mobility (e.g. cross-slip and climb) and more wavy slip at $650^{\circ} \mathrm{C}$ which may accelerate Stage II crack growth processes. The increase in dislocation mobility at elevated temperatures will also lead to reduction in the yield strengths of the materials. 
This will also contribute to increased crack growth rates due to an increase in the crack tip opening for a given stress level. However a reduction of $20 \%$ in $\sigma_{\mathrm{y}}$ due to increased temperature will only produce an increase of $\sim 50 \%$ in apparent $d a / d N$, so the increase in $d a / d N$ from room temperature to $650^{\circ} \mathrm{C}$ cannot purely be due to yield strength variation alone. The difference in crack growth rates is also unlikely to be linked to increased oxidation effects due to the effective absence of environment effects at $650^{\circ} \mathrm{C}$ in vacuum compared to room temperature in air, although the change in environment may also have affected slip behaviour. Generally, vacuum environments are considered to promote slip reversibility and hence enhance heterogeneous slip processes $c f$. tests in similar conditions in air $[16,17]$. In this case it therefore appears that the increase in temperature has significantly affected slip character (despite the vacuum conditions) and this is the principal cause of the acceleration in crack growth rates

\subsection{Effects of microstructure on room temperature fatigue threshold and long crack growth}

5.5.1. Slip character effects. Intrinsic crack growth resistance in both Stage I and II is controlled by slip character but there are various opposing effects of slip character on intrinsic crack growth resistance. Firstly, highly planar slip has been associated with more intense impingement of slip bands at grain boundaries due to greater pile-up of dislocations in the slip bands. Thus dislocation sources are able to nucleate more easily in the adjacent grain and crack growth processes continue into the next grain. Under such circumstances, grain boundaries are less effective as crack growth barriers, resulting in poorer crack growth resistance. Secondly, intense planar slip, where dislocations are confined and move to and fro in relatively few slip bands, gives greater reversibility of slip and increased intrinsic crack growth resistance. Finally, highly planar slip has also been associated with more tortuous crack paths compared to those produced by homogeneous slip. Large crack path deflections due to intense slip bands extending the diameter of the grain may give rise to crack tip shielding and possible roughness-induced crack closure, i.e. higher extrinsic crack growth resistance. The crack closure contribution to crack growth resistance is therefore also likely to be influenced by grain size.

Planarity of slip could also be linked to the degree of faceting observed during stage I crack growth. The current study has found the most distinct crystallographic facets in U720Li with predominantly micro-facets and a low proportion of large facets observed in U720Li-LG and U720Li-LP, however corresponding short crack results [7] showed the most distinct crystallographic facets during early stage I crack growth in U720Li-LG, followed by U720Li while U720Li-LP showed the least crystallographic crack growth amongst the materials. This difference between short crack and long crack fractography may be related to the stress state and/or constraints of the long crack growth 
geometry. The constraints of neighbouring slip systems from adjacent grains along the crack front will differ between the two cases. In the long crack case, the requirement for slip systems to conform with those from adjacent grains along a multi-grained crack front may result in slip bands not being able to extend across the entire grain diameter compared to the short crack situation where there are less grains along the crack front, reducing the requirements of slip system conformity. It seems likely therefore that the short crack Stage I crack growth fractography is most representative of slip character, with the most planar slip character seen therefore in U720Li-LG, followed by a lower degree of planar slip character in U720Li and the least planar slip character in U720Li-LP.

5.5.2. Grain size effects. Grain size also has two possible opposing effects on crack growth resistance. A fine-grained material has more grain boundaries acting as barriers to dislocation motion and crack growth, providing better crack growth resistance. However, grain size also influences extrinsic crack growth resistance if planar slip processes are in operation. Larger grains have been associated with a more tortuous crack path, giving increased crack tip deflection and shielding in addition to increased crack closure during long crack growth, all of which will result in better extrinsic crack growth resistance. However the extension of a planar slip band across a large grain may be affected by the requirement to conform with slip systems of adjacent grains (as discussed above) hence it may be easier for slip to extend across the entire grain diameter giving distinct crystallographic facets on the fracture surface for a finer grained material (as observed when comparing U720Li and U720Li-LG)

Extrinsic crack growth resistance contributions are expected to arise from crack tip shielding and crack closure. Typical crack tip deflections may be most significant when defect size is small, i.e. during short crack growth. Assessment of crack deflections on crack growth driving force indicates that local crack tip deflections observed in long crack tests in the current study gave only marginal fluctuations (up to $\sim 20 \%$ decreases $c f$. global values) in crack growth driving force $\left(\Delta K_{\text {eff }}\right.$ ) at the crack tip. This suggests that any extrinsic contribution to crack growth resistance is likely to arise primarily from crack closure. Crack closure measurements were not made in the current study, hence closure levels can only be qualitatively estimated from the fretting damage associated with crack closure on the near-threshold crack growth fracture surfaces as shown in Figures 4-6. Limited evidence of fretting damage was noted on the U720Li fracture surface, suggesting minimal crack closure, whereas U720Li-LP and U720Li-LG respectively showed increasing levels of fretting damage, suggesting some degree of closure in U720Li-LP and a higher degree of closure in U720Li-LG. The degree of fretting damage in the materials appear to suggest that closure is not controlled so much by the degree of faceting but is governed more by the grain size of the materials. The fracture surface of U720Li in the near-threshold crack growth region is not only highly crystallographic, but is more consistently 
tortuous (i.e. it has the greatest ratio of actual to projected crack path) but appears to have the least closure. The occasional large facets produced in U720Li-LP and even larger ones seen in U720Li-LG therefore appear more critical in producing closure. This is in line with findings of several recent modelling papers assessing the role of defect asperities in closure [18].

During near-threshold stage I crack growth, U720Li-LP showed the poorest crack growth resistance as evidenced from the lowest $\Delta K_{t h}$ compared to other materials. U720Li and U720Li-LG show approximately similar $\Delta K_{t h}$, suggesting similar stage I crack growth resistance in these materials. Overall, the fractographic observations and crack growth results indicate that fatigue threshold and near-threshold stage I crack growth resistance is governed by varying balances of intrinsic and extrinsic crack growth resistance contributions. Intrinsic crack growth resistance contributions are considered therefore to arise from reversibility of slip in U720Li and U720Li-LG, and the combination of reduced intense impingement of slip bands at grain boundaries and some degree of reversible slip in U720Li-LP. Extrinsic crack growth resistance contributions arise from crack closure during the tests.

U720Li-LG shows slightly higher fatigue threshold and near-threshold stage I crack growth resistance compared to U720Li as both U720Li and U720Li-LG show similar degrees of apparent slip reversibility but U720Li-LG appears to have a higher degree of crack closure, i.e. a higher extrinsic crack growth resistance contribution. U720Li-LP shows the lowest overall fatigue threshold and stage I crack growth resistance compared to other materials due to a lower intrinsic crack growth resistance contribution in U720Li-LP compared to other materials which was not compensated for by the extrinsic crack growth resistance contribution from crack closure. The lower intrinsic crack growth resistance contribution in U720Li-LP is attributed to a combination of precipitate cutting and bowing mechanisms in the material.

In considering stage II crack growth at higher $\Delta \mathrm{K}$ levels, the influence of local microstructural effects and extrinsic effects on crack growth resistance is expected to diminish. Any effects of microstructure on stage II crack growth are generally expected to be governed more by the intrinsic crack growth resistance related to reversibility of slip. The crack growth rates of U720Li and U720Li-LG in this regime therefore indicate relatively similar intrinsic crack growth resistance due to slip character and its effect on damage accumulation. In U720Li-LP, the intrinsic crack growth resistance contribution due to more homogeneous slip (although some degree of reversible slip is expected due to precipitate cutting) is only marginally lower than for U720Li and U720Li-LG. 
Overall, the deformation behaviour of the materials considered here, whether homogeneous or heterogeneous, appears to give approximately equivalent intrinsic crack growth resistance at room temperature due to the respective effects of each deformation behaviour on intrinsic crack growth resistance. Greater planarity of slip has more effect on improved extrinsic crack growth resistance contributions, such as crack shielding and closure.

\subsection{SUMMARY AND CONCLUSIONS}

Room temperature long crack fatigue tests have been carried out on U720Li and microstructural variants of U720Li, i.e. U720Li-LG and U720Li-LP. Fracture surfaces were noted to be crystallographic during near-threshold crack growth, indicative of stage I crack growth. U720Li shows distinct crystallographic facets on the fracture surface while U720Li-LG and U720Li-LP exhibit mostly microfacets and a lower proportion of large facets. As $\Delta K$ increases, crack growth follows a progressively less crystallographic and flatter transgranular stage II crack path. U720Li-LG demonstrated the highest $\Delta K_{t h}$ amongst the materials, with U720Li showing intermediate $\Delta K_{t h}$ while U720Li-LP shows the lowest $\Delta K_{t h}$. U720Li-LP was also noted to show higher crack growth rates in the near-threshold regime while at high $\Delta K$ the crack growth rate of U720Li-LP is marginally higher compared to the other two materials. Crack growth rates of U720Li and U720Li-LG were relatively similar both in the near-threshold and high $\Delta K$ regime. The result of this study indicates larger grains to be beneficial for room temperature fatigue threshold and crack growth resistance due to increased extrinsic crack growth resistance contributions from crack closure. Differences in heterogeneity of deformation behaviour in this set of material variants do not appear to give significant difference in intrinsic crack growth resistance at room temperature due to the respective effects of each deformation behaviour on intrinsic crack growth resistance.

\section{ACKNOWLEDGEMENTS}

Financial support from QinetiQ Farnborough, the School of Engineering Sciences and the Overseas Research Scholarship (ORS) scheme administered by the Committee of Vice-Chancellors and Principals, United Kingdom is gratefully acknowledged. Dr Mike Henderson, Mr Ian Wilcock, Prof George Harrison, Dr. Mike Winstone and Dr Jeff Brooks are thanked for many useful discussions of this (and related) work.

\section{REFERENCES}

${ }^{1}$ Sadananda K., Shahinian P. Proc. Int. Conf. on Corrosion of Nickel-Base Alloys, Cincinnati, Ohio, USA, 23-25 Oct. 1984, American Society for Metals,101-115, 1985. 
${ }^{2}$ Nikbin K.M., Webster G.A. Proceedings 2 nd International Conference on Creep and Fracture of Engineering Materials and Structures, Swansea, U.K., 1-6 Apr. 1984, Pineridge Press Ltd., 2, 10911104, 1984.

${ }^{3}$ Ritchie, R.O. and Suresh, S. (1981) Metallurgical Transactions 13A 937-40

${ }^{4}$ Tucker A., Henderson M. B., Wilkinson A. J., Hide N.J., and Reed P.A.S. Materials Science \&

Technology, 18 (3) 2002, 349-353.

${ }^{5}$ Everitt, S., Starink, M.J., Pang, H.T., Wilcock, I.M., Henderson, M.B. and Reed, P.A.S. submitted to Materials Science \& Technology March 2007

${ }^{6}$ Hide N.J., Henderson M.B., Reed P.A.S. Proc. 9th International Symposium on Superalloys, Seven Springs, PA, USA (2000), Minerals, Metals and Materials Society/AIME, Superalloys 2000 (2000) 495-503.

${ }^{7}$ Pang, H.T. and Reed P.A.S. submitted to ? March 2007

${ }^{8}$ H.T. Pang and P.A.S. Reed Materials Science and Engineering A, 2007, 448, 67-79

${ }^{9}$ Bilby, B.A., Cardew, G.E., Howard, I.C. (1977) In Fracture 1977 (ed. D.M.R. Taplin) 3 197-200, New York, Pergamon Press

${ }^{10}$ D. Hunt, University of Cambridge, 1998, Private communication

${ }^{11}$ Jackson, M.P. and Reed, R.C. Materials Science and Engineering A (1999) 259 85-97

${ }^{12}$ Mao, J. Chang, K.M. Yang, W., Ray, K., Vase, S.P., Furrer, D.U. (2001) Metallurgical and Materials Transactions A 32 2441-2452

${ }^{13}$ Lerch B.A., Jayaraman, N., Antolovich, S.D. Materials Science and Engineering A 66 151-166 (1984)

${ }^{14}$ Subramanya Sarma V., Sundaraman, M. Padmanabhan, K.A. Materials Science and Technology 14, 669-675 (1998)

${ }^{15}$ Irwin, G.R. (1960) In Proc. of Seventh Sagamore Ordnance Materials Conference IV, 63-78 New York, Syracuse University

${ }^{16}$ Duquette, D.J. \& Gell, M. Met. Trans 2: 1325-1331 (1971).

${ }^{17}$ Reed, P.A.S., Wu, X.D. and Sinclair, I., Met \& Mat.Trans, 31A: 2000, 109-123

${ }^{18}$ Kamp N, Parry MR, Singh KD, Sinclair I, Acta Materialia 52 (2): 343-353 2004 